\title{
One Dimensional Hybrid-Vlasov Simulation of a Hall Thruster
}

\author{
Kentaro Hara * and Iain D. Boyd ${ }^{\dagger}$ \\ University of Michigan, Ann Arbor, MI 48109-2140 \\ Vladimir I. Kolobov $\ddagger$ \\ CFD Research Corporation, Huntsville, AL 35805
}

\begin{abstract}
Velocity distribution functions (VDFs) in a Hall thruster are known to be non-Maxwellian. For instance, the acceleration and ionization occur simultaneously inside the channel so that the ions have a bi-Maxwellian VDF. In order to model the behavior of the plasma, two main methods have been developed: fluid and particle methods. Fluid models assume near-Maxwellian VDFs and particle methods, although nonequilibrium phenomena are captured, suffer from statistical noise. By comparison, a direct simulation method which solves the Vlasov equation coupled with collision terms can achieve better resolution of VDFs. In this study, a one-dimensional direct Vlasov simulation using a bounded upwind scheme is developed and applied for a plasma simulation in a Hall thruster channel. Results obtained from the hybrid-Vlasov simulation show good agreement with hybrid-PIC results and experimental data. Low frequency plasma oscillations, often referred to as the breathing mode, are observed. Using the Vlasov simulation, an improved resolution of VDFs with less statistical noise is obtained in comparison to the particle simulation.
\end{abstract}

\section{Nomenclature}

Subscript

$e \quad$ Electron

$i \quad$ Ion

$n \quad$ Neutral atom

Mass, $\mathrm{kg}$

Collision term

Time, $\mathrm{s}$

Velocity, $\mathrm{m} / \mathrm{s}$

Magnetic field, G

Elementary charge, $\mathrm{C}$

Electric field, V/m

Velocity distribution function

Discharge current, A

Anode mass flow rate, $\mathrm{kg} / \mathrm{s}$

Number density, $\mathrm{m}^{-3}$

Physical space, $\mathrm{m}$

Mean velocity, $\mathrm{m} / \mathrm{s}$

Discharge voltage, $\mathrm{V}$

Mean electron energy, eV

Electron mobility, $\mathrm{m}^{2} /(\mathrm{V} \cdot \mathrm{s})$

Collision frequency, $\mathrm{s}^{-1}$

* Graduate Student, Department of Aerospace Engineering, Student Member, AIAA.

${ }^{\dagger}$ Professor, Department of Aerospace Engineering, Fellow, AIAA.

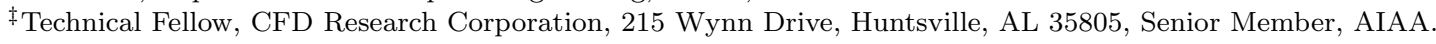




\section{Introduction}

The partially magnetized plasma in Hall thrusters is known to be in a nonequilibrium state. For example, the regions of ionization and acceleration overlap, which yields a bi-Maxwellian velocity distribution function (VDF) for ions. In addition, the electrons experience complex processes during thruster operation. High energy electrons are more likely to collide with the channel walls yet contribute to ionization. The VDFs of electrons are expected to deviate from the equilibrium Maxwellian form.

Two methods have mainly been used for plasma simulation. A fluid approach is based on solving the conservation equations of mass, momentum, and energy, and assumes the VDFs are close to Maxwellian. ${ }^{1,2}$ This method has been well developed and relatively efficient in terms of computational cost. The other approach involves particle methods that are able to capture nonequilibrium phenomena. ${ }^{3,4}$ Due to the use of macroparticles, particle methods suffer from numerical noise and the inability to resolve the high-energy tail of the electron VDFs that can contribute to inelastic collisions. By comparison, the Boltzmann equation, or the Vlasov equation coupled with collision terms, can be solved to obtain the VDFs. ${ }^{5}$ The primary purpose of the present investigation is to develop a direct Vlasov simulation and assess its capability for modeling the low temperature plasmas in a Hall thruster.

In this paper, a direct simulation method is used to solve the Vlasov equation coupled with collision terms. VDFs in discretized phase space are directly computed using a direct Vlasov simulation. Sections II and III will describe the thruster and the plasma model used in the current simulation, resepectively. In Section IV, a bounded upwind scheme is developed for the direct Vlasov simulation and the numerical setup is described. Finally, in Section V, results obtained from the hybrid-Vlasov simulation are presented and discussed.

\section{SPT-100 Thruster}

The Stationary plasma thruster (SPT) is a magnetic layer type Hall thruster. The configuration of the SPT-100 thruster considered here is summarized in Table 1. Hall current is produced in the azimuthal direction by the interaction of the radial magnetic field and the axial electric field. Electrons are trapped and heated near the region of maximum magnetic field. Neutral atoms injected from the anode are ionized mainly by the trapped electrons and accelerated by the electric field as soon as ions are generated. The channel length and width are designed to allow electrons to gyrate along the magnetic field whereas ions are accelerated toward the exit without being magnetized. Due to the curvature of the electric field, some amount of ions collide with channel walls that results in wall erosion, which limits the lifetime of Hall thrusters. Since the model is one-dimensional, only the radial magnetic field and axial electric field are taken into consideration.

Table 1. SPT-100 configuration

\begin{tabular}{|rc|c|}
\hline Axial length & $L$ & $4 \mathrm{~cm}$ \\
Mass flow rate & $\dot{m}$ & $5 \mathrm{mg} / \mathrm{s}$ \\
Discharge voltage & $V_{d}$ & $300 \mathrm{~V}$ \\
Discharge current & $I_{d}$ & $4.5 \mathrm{~A}$ \\
Maximum Magnetic field & $B_{0}$ & $160 \mathrm{G}$ \\
\hline
\end{tabular}

Garrigues et al. used two different magnetic field profiles and showed that the mean discharge current is similar but the amplitude and shape of the oscillations vary significantly. ${ }^{6}$ Since the main goal of this paper is developing the Vlasov simulation and demonstrating its capability for application to a Hall thruster, the effect of the curvature of magnetic field distribution is not considered due to the one-dimensional assumption. 


\section{Plasma model}

The basis of the hybrid-Vlasov simulation is the formulation proposed by Boeuf and Garrigues. ${ }^{7}$ This approach consists of a Vlasov solver for heavy particles such as ions and neutral atoms, and a fluid model for electrons. In our model, the neutral atoms are modeled by using a Vlasov solver rather than assuming a continuum flow. Due to their much smaller time scale, the electrons are assumed to be in a state of equilibrium compared to the heavy species. The fluid model greatly reduces the computational cost in comparison to applying a direct Vlasov simulation for electrons. In the present model, collision mechanisms such as single-charge ionization and charge exchange are also included for heavy species.

\section{A. Vlasov equation coupled with collision terms}

The Vlasov equation is often referred to as the collisionless Boltzmann equation in plasma physics. Unlike high temperature plasmas, collisions play an important role for low temperature plasmas. The Vlasov equation coupled with collision terms can be written as,

$$
\frac{\partial f_{s}}{\partial t}+\vec{v} \cdot \frac{\partial f_{s}}{\partial \vec{x}}+\frac{q_{s}}{m_{s}}(\vec{E}+\vec{v} \times \vec{B}) \frac{\partial f_{s}}{\partial \vec{v}}=S_{s} .
$$

where $q_{s}, m_{s}, f_{s}$, and $S_{s}$ are the charge, mass, VDF, and collision term of species, $s$, respectively. The collision term and VDF are functions of physical space, $\vec{x}$, velocity, $\vec{v}$, and time, $t$. Macroscopic quantities can be obtained by evaluating moments of the VDFs. The number density, mean velocity, and mean energy are obtained from

$$
\begin{aligned}
n(\vec{x}, t) & =\int_{-\infty}^{\infty} f(\vec{x}, \vec{v}, t) d \vec{v} \\
\vec{u}(\vec{x}, t) & =\int_{-\infty}^{\infty} \vec{v} \cdot \hat{f}(\vec{x}, \vec{v}, t) d \vec{v} \\
\epsilon(\vec{x}, t) & =\int_{-\infty}^{\infty} \frac{1}{2} m v^{2} \cdot \hat{f}(\vec{x}, \vec{v}, t) d \vec{v}
\end{aligned}
$$

where $\hat{f}$ is the normalized VDF: $\hat{f}=f / n$. Note that the conventional fluid conservation equations can be derived by taking moments of the collisional invariants in Equation 1 and by assuming the VDFs are Maxwellian.

\section{Ion Vlasov equation}

The present one-dimensional simulation includes one dimension in both space and velocity (1D1V). Additionally, the Lorentz force can be neglected for ions in the channel of a Hall thruster since the magnetic field is chosen so that they are relatively non-magnetized. The one-dimensional ion transport equation is given by

$$
\frac{\partial f_{i}}{\partial t}+v_{x} \frac{\partial f_{i}}{\partial x}+\frac{e E}{m_{i}} \frac{\partial f_{i}}{\partial v_{x}}=S_{i}
$$

\section{Neutral atom Vlasov equation}

Neutral atoms are not in the continuum regime based on the Knudsen number experienced in a Hall thruster. Thus, a kinetic Vlasov approach can also be used in order to model neutral atoms. Due to the absence of any external force, the neutral transport equation is given by

$$
\frac{\partial f_{n}}{\partial t}+v_{x} \frac{\partial f_{n}}{\partial x}=S_{n}
$$

\section{Collision terms}

Depending on the processes that are accounted for, the collision terms can be calculated as a sum of each collision process. For heavy species, single-charge ionization and charge exchange collisions are included. 
The ion and neutral collision terms are given by

$$
\begin{aligned}
& S_{i}\left(x, v_{x}, t\right)=\dot{n}^{\mathrm{ION}} \hat{f}_{n}\left(v_{x}\right)+\dot{n}^{\mathrm{CEX}}\left\{\hat{f}_{n}\left(v_{x}\right)-\hat{f}_{i}\left(v_{x}\right)\right\} \\
& S_{n}\left(x, v_{x}, t\right)=-\dot{n}^{\mathrm{ION}} \hat{f}_{n}\left(v_{x}\right)-\dot{n}^{\mathrm{CEX}}\left\{\hat{f}_{n}\left(v_{x}\right)-\hat{f}_{i}\left(v_{x}\right)\right\} .
\end{aligned}
$$

where $\dot{n}^{\mathrm{ION}}$ and $\dot{n}^{\mathrm{CEX}}$ are the collision rates due to ionization and CEX, and $\hat{f}_{n}\left(v_{x}\right)$ and $\hat{f}_{i}\left(v_{x}\right)$ are the normalized VDFs for neutral atoms and ions, respectively. For instance, for each ionization event, an ion is generated and a neutral atom is deleted. The generated ion has the same location and velocity as the deleted atom so that the change in VDFs due to ionization is proportional to the VDFs of neutral atoms. Note that $S_{i}=-S_{n}$ since the number of particles must be conserved in collisions between neutral atoms and ions. The magnitude of the change in VDFs due to collisions for neutral atoms must equal that for ions.

The ionization rate is calculated by assuming a Maxwellian VDF for collisions between electrons and neutral atoms

$$
\dot{n}^{\mathrm{ION}}=n_{n} n_{e} \iiint \hat{f}_{n}\left(v_{n}\right) \hat{f}_{e}\left(v_{e}\right) g \sigma^{\mathrm{ION}}(g) d v_{n} d v_{e} d \Omega=n_{n} n_{i} \xi^{i}\left(T_{e}\right)
$$

where $g$ is the magnitude of the relative velocity, $\sigma(g)$ is the differential cross section, and $\Omega$ is the solid angle of a collision. Assuming neutral atoms are much slower than electrons, the ionization rate becomes a function of electron temperature, $T_{e}$.

In a charge exchange (CEX) collision, a fast ion collides with a slow neutral atom which results in a slow ion and a fast neutral atom. Assuming that neutral atoms follow a Maxwellian distribution and are much slower than ions, the rate of CEX is calculated using the ion VDF

$$
\dot{n}^{\mathrm{CEX}}=n_{n} n_{i} \iiint \hat{f}_{n}\left(v_{n}\right) \hat{f}_{i}\left(v_{i}\right) g \sigma^{\mathrm{CEX}}(g) d v_{n} d v_{i} d \Omega .
$$

For the cross section of ionization, the data of Puech and Mizzi ${ }^{8}$ are used, and the cross section for CEX employs the expression proposed by Pullins et al. ${ }^{9}$

\section{B. Fluid model}

A fluid model is used for electrons, which assumes an equilibrium state, since the characteristic time scales of electrons are much smaller than those of ions. We assume that the electron temperature is much larger than the ion and neutral temperatures. The transfer of momentum and energy is approximated by a Krook collision operator using transfer frequency. A quasi-neutral assumption is used so that the number density of ions equals that of electrons and the electric field can be obtained from the momentum transfer equation. The steady state momentum and energy equations for the electrons are

$$
\begin{aligned}
0 & =-n \mu E+n \bar{u}_{e} \\
\frac{5}{3} \frac{\partial \bar{\epsilon}}{\partial x} & =-e E+\frac{\nu_{\epsilon}}{\bar{u}_{e}} \bar{\epsilon}
\end{aligned}
$$

where $n$ is the plasma density (equal to ion number density, $n_{i}$, and electron number density, $n_{e}$, due to the quasi-neutrality), and $\nu_{\epsilon}$ is the total electron energy transfer frequency. Note that inertia, diffusion, and pressure terms are neglected in the momentum equation. The energy transport equation is simplified by neglecting the thermal flux term and assuming the electron thermal energy is much larger than the electron drift energy. The total current or discharge current is calculated by the sum of ion and electron current

$$
I_{T}=I_{i}-I_{e}
$$

where $I_{T}, I_{i}$, and $I_{e}$ are the total current, ion current, and electron current, respectively.

\section{Electron mobility}

The electric field is calculated by Equations 8a and 9 using the fundamental relation that the total integrated electric field from cathode to anode equals the discharge voltage, $V_{d}$. Electron mobility across the magnetic 
field is modeled using the classical description. Therefore, the transverse electron mobility in the cross-field direction can be written as

$$
\mu=\frac{e}{m_{e} \nu_{m}} \frac{1}{1+\omega_{B}^{2} / \nu_{m}^{2}}
$$

where $\omega_{B}$ is the electron cyclotron frequency and $\nu_{m}$ is the total electron momentum transfer frequency.

\section{Collision frequency}

The collision frequencies used in the present simulation are based on the model used by Boeuf and Garrigues ${ }^{7}$ and are summerized here. The total electron momentum transfer frequency in Equation 10 can be written as a sum of contributions from electron-neutral collisions, $\nu_{m}^{\mathrm{e}-\mathrm{n}}$, and electron-wall collisions, $\nu_{m}^{\text {wall }}$.

$$
\begin{aligned}
\nu_{m}^{\mathrm{e}-\mathrm{n}} & =n_{n} k_{m} \\
\nu_{m}^{\text {wall }} & =\alpha \times 10^{7}
\end{aligned}
$$

where $k_{m}$ is the electron momentum exchange rate. $k_{m}$ and $\alpha$ are constant parameters which can be adjusted. In this study, we set those parameters as $k_{m}=2.5 \times 10^{-13} \mathrm{~m}^{3} \mathrm{~s}^{-1}$ and $\alpha=0.2$. Similarly, the total electron energy transfer frequency in Equation $8 \mathrm{~b}$ can be modeled as a sum of contributions from electron-neutral collisions, $\nu_{\epsilon}^{\mathrm{e}-\mathrm{n}}$, and electron-wall collisions, $\nu_{\epsilon}^{\text {wall }}$, which are given by

$$
\begin{aligned}
\nu_{\epsilon}^{\mathrm{e}-\mathrm{n}} & =n_{n}\left(\xi^{i}(\bar{\epsilon})+\xi^{e x}(\bar{\epsilon})\right) \\
\nu_{\epsilon}^{\text {wall }} & =\alpha \times 10^{7} \exp (-U / \bar{\epsilon})
\end{aligned}
$$

where $\xi^{i}(\bar{\epsilon})$ and $\xi^{e x}(\bar{\epsilon})$ are the rates of ionization and excitation, respectively, and $U$ is a parameter taken to be equal to $20 \mathrm{eV}$. Figure 1 shows the ionization and excitation rates that are used by Boeuf and Garrigues ${ }^{7}$ using the cross sections of Puech and Mizzi. ${ }^{8}$ The ionization rate is also used for the collision terms of ions and neutral atoms in Equation 6. In addition, the electron mean energy is related to electron temperature:

$$
T_{e}=\frac{2}{3} \bar{\epsilon}
$$

where $T_{e}$ is the electron temperature in $\mathrm{eV}$.

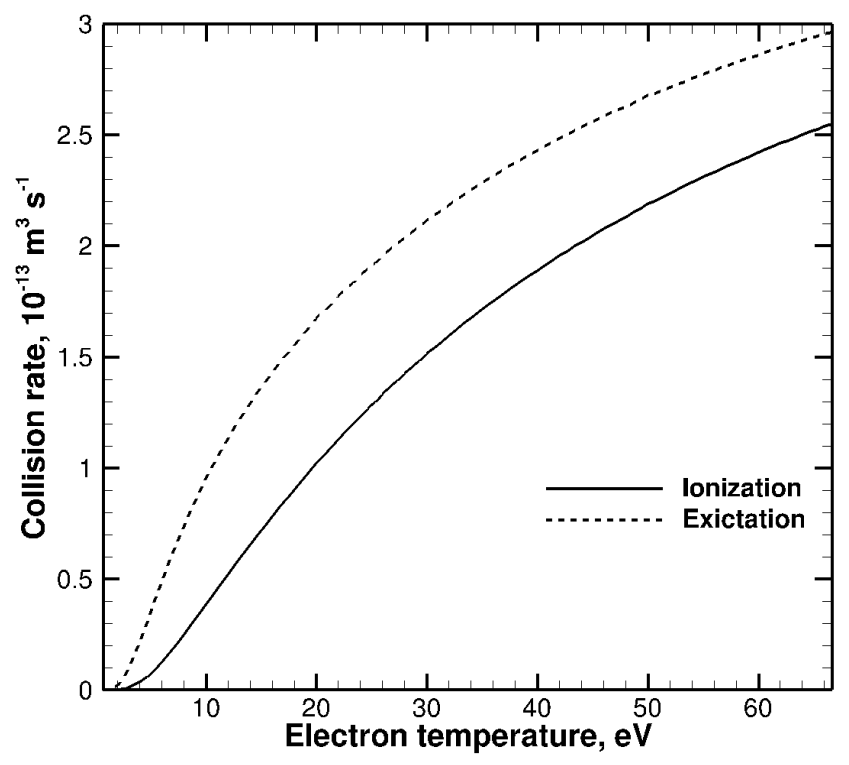

Figure 1. Ionization and excitation rate 


\section{1D hybrid-Vlasov simulation}

The one-dimensional hybrid-Vlasov simulation is used to model the partially magnetized plasma inside the channel of a Hall thruster. Our primary purpose is to apply the direct Vlasov simulation for Hall thruster simulation and investigate its capability. Analysis of the electron fluid model is not presented since the effects of the models and parameters of electron mobility and other collision frequencies are shown in several papers. ${ }^{10,11}$

\section{A. Vlasov Simulation}

Boeuf and Garrigues solved the Vlasov simulation for heavy species using an upwind scheme. ${ }^{7}$ According to their paper, the ion VDFs show obvious numerical diffusion effects in comparison to the Monte Carlo method. We develop a direct Vlasov simulation which has less dissipation, or higher order of accuracy, while preserving the positivity of VDFs.

A direct Vlasov simulation solves the time-dependent Vlasov equation coupled with collision terms as shown in Equations 3 and 4. Two types of Vlasov solvers have been mainly developed. One is a semiLagrangian scheme employing dimensional splitting, ${ }^{12}$ which divides the Vlasov equation into two advection equations: $\partial_{t} f+v \partial_{x} f=0$ and $\partial_{t} f+a \partial_{v} f=0$ where $v$ is the velocity, $a$ is the acceleration, and $\partial_{t}, \partial_{x}$ and $\partial_{v}$ are the time, space, and velocity derivatives, respectively. The spatial derivatives are evaluated with a finite-differencing scheme using high-order interpolations such as cubic-spline interpolation. ${ }^{13}$ The other type of solver is an Eulerian grid method ${ }^{14}$ which does not use any dimensional splitting so that boundary conditions are easier to set. In contrast to semi-Lagrangian schemes, an Eulerian Vlasov solver is a finitevolume type scheme which inherently satisfies conservation. The downside is that a smaller time step is necessary due to stability, which results in an increase of computational cost. ${ }^{15}$ In our model, the Corner Transport Upwind (CTU) scheme ${ }^{14}$ is used. This scheme is second order accurate with a larger time step than a first order upwind scheme. ${ }^{16}$

$$
f(\vec{x}, \vec{v}, t) \geq 0
$$

A requirement with deterministic Vlasov solvers is that the distribution function must remain positive. One difficulty of multidimensional calculations is that unnecessary oscillations are usually generated. For unsteady simulations, especially in a partially magnetized plasma that contains several frequencies of physical oscillations, ${ }^{17}$ any numerical fluctuations make the simulation inaccurate. Furthermore, unphysical negative number densities may be computed and the simulation becomes unstable.

In order to statisy the physical boundedness, a bounded upwind scheme that imposes a global physical constraint (Equation 13) on the VDFs is developed. It is based on the model proposed by Herrmann et al. that prevents unphysical solutiuons for the mixture fraction in Large Eddy Simulation. ${ }^{18}$ Here, we call the new Vlasov algorithm the Bounded-CTU scheme. The algorithm is described in Table 2.

Table 2. Bounded upwind scheme

for all control volumes $V:$ calculate $\phi_{i}^{*}$ from second order scheme.

for all control volumes $V:$ if $\phi^{*} \leq 0: \phi_{i}^{n+1}$ is calculated from first order upwind scheme.

else: $\phi_{i}^{n+1}=\phi_{i}^{*}$

For any linear advection equation, numerical oscillations will be produced using a scheme that is higher than first order according to Godunov's theorem. The use of a limiter allows us to suppress oscillations. However, for multi-dimensional simulations, numerical oscillations are still difficult to be suppressed. A first-order upwind scheme, which is a monotonicity preserving scheme, is used for the cells in which any unphysical value (i.e. negative VDFs) is produced by the higher order scheme. For the other cells where physical boundedness is satisfied (Equation 13), the value obtained using the higher order scheme is updated without any modification. Thus, the scheme maintains a global second order of accuracy.

In addition, the stability condition is the most restrictive criterion to satisfy for the computation. The second-order corner transport upwind scheme is an improved scheme to achieve the following stability condition.

$$
\max \left(\frac{v \Delta t}{\Delta x}, \frac{a \Delta t}{\Delta v}\right) \leq 1
$$




\section{B. Boundary Conditions and discretization}

Boundary conditions of the Vlasov simulation play an important role. Reid concluded that the mean inlet velocity of neutral atoms entering a Hall thruster channel has a square root dependence on the anode temperature. ${ }^{19}$ For the anode boundary condition of neutral atoms, we applied a half-Maxwellian VDF with zero mean velocity and a temperature of $750 \mathrm{~K}$. The mean velocity of a half-Maxwellian is calculated as

$$
\bar{u}_{n}=\sqrt{\frac{2 k_{B} T_{n}}{\pi m_{n}}}
$$

where $k_{B}$ is the Boltzmann constant and $T_{n}$ is the neutral temperature. This agrees with the observation by Reid. It is assumed that the neutral temperature inside the channel is equal to the wall temperature, $T_{n}=750 \mathrm{~K}$. The inlet mean velocity of neutral atoms is $173 \mathrm{~m} / \mathrm{s}$. Additionally, the inlet density of neutral atoms is calculated by the mass flow rate and the inlet velocity.

In order to capture the unsteady physics of Hall thrusters, where strong plasma oscillations may occur, velocity space should be chosen properly. The mean properties are calculated by integrating the moments of VDFs inside the domain and will be underestimated due to truncating VDFs if the domain is too small. We choose the maximum and minimum velocity to be $90000 \mathrm{~m} / \mathrm{s}$ and $-15000 \mathrm{~m} / \mathrm{s}$, respectively. Most importantly, the velocity bins must be chosen carefully in order to discretize the VDFs accurately. The mean velocity of ions is approximately $200 \mathrm{~m} / \mathrm{s}$ at the anode and $20,000 \mathrm{~m} / \mathrm{s}$ at the channel exit. While satisfying the stability condition, the phase space must be discretized finely enough such that the VDFs are well resolved. For the present case, it is found that $\Delta v \leq 300$ is required.

The mean electron energy at the channel exit is set to $10 \mathrm{eV}$ (the electron temperature is $6.7 \mathrm{eV}$ ). A fourth-order Runge-Kutta method is used to calculate the mean electron energy. The potential is set to 0 $\mathrm{V}$ at the channel exit and the anode potential is equal to the discharge voltage. Note that the anode sheath and the plume are neglected in this model.

\section{Results}

The 1D hybrid-Vlasov simulation is performed and the results are compared with those obtained from a 1D hybrid-PIC simulation and experiments. Both simulations do not reach a steady state due to the dynamic effect of the partially magnetized plasma in Hall thrusters. In order to investigate the capability of the hybrid-Vlasov simulation, time averaged results and time resolved results are investigated.

For the Vlasov simulation, the time step size used for the xenon particles is $1 \times 10^{-9}$ seconds and the cell size is $\left[4 \times 10^{-4} \mathrm{~m}, 250 \mathrm{~m} / \mathrm{s}\right]$. The phase space is divided into $100 \times 400$ cells in the physical space and velocity space, respectively. The time step and phase space discretization are chosen to satisfy the stability condition in Equation 14.

The hybrid-PIC simulation developed by Boeuf and Garrigues ${ }^{7,20}$ uses a time step of $1 \times 10^{-8}$ seconds in order to satisfy the CFL condition in the physical space. The number of macroparticles used in the simulation is approximately 300,000 particles after the effect of macroparticle counts is investigated. The total number of macroparticles is fixed instead of keeping it constant in each cell. The number of cells in the physical space is fixed at 100. For the hybrid-PIC simulation, the continuity equation is solved for neutral atoms with constant speed, which is set to $173 \mathrm{~m} / \mathrm{s}$ in the present simulation.

\section{A. Time averaged plasma properties}

In order to assess the 1D hybrid-Vlasov simulation, macroscopic results are compared with the results obtained from the 1D hybrid-PIC simulation and with experimental data. The same fluid model described in Section III-B is used for both hybrid-Vlasov and hybrid-PIC methods. The effect of CEX is also investigated. The time averaged macroscopic results are averaged over several oscillation cycles.

\section{Plasma properties}

As shown in Figure 2, time averaged plasma properties are obtained from the simulation. It can be seen that there are mainly three regions in the channel of a Hall thruster: diffusion, ionization, and acceleration zones. Starting on the left at the anode, there is a relatively flat region in which little ionization occurs. This diffusion zone contributes to a stable source of neutral atoms into the channel. Secondly, ions are generated 
and accumulated in the ionization region. The ions accumulate because of the small electric field so that they are not accelerated out of the channel. The rate of ionization depends on the electron temperature and the number densities of neutral atoms and electrons so that the peak of ionization lies between the peaks of ion number density and electron temperature. Finally, on the right near the channel exit, in the acceleration region, ions are accelerated due to the steep electric field. The mechanism of a steep potential drop can be explained from the large resistance due to the trapped electrons in this region. The magnetic field is largest at the channel exit and the axial electron mobility is small so that a larger electric field is required to drive the same current.

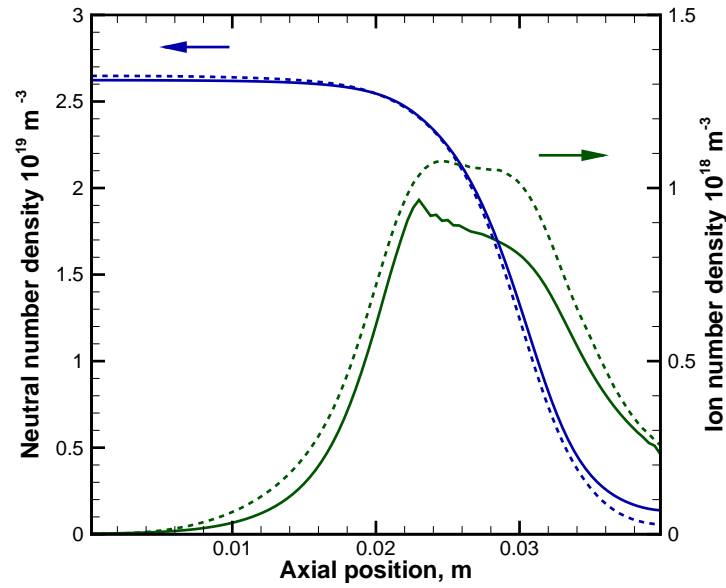

(a) Heavy species

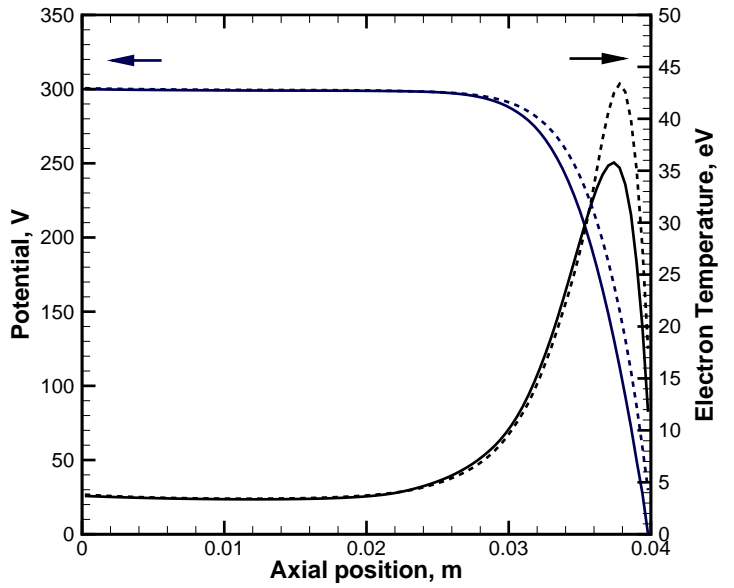

(b) Electrons

Figure 2. Time averaged results of macroscopic parameters. Solid lines: Hybrid-Vlasov simulation, Dashed lines: Hybrid-PIC simulation

The results obtained from both simulations show good agreement. However, the potential drop near the channel exit is slightly steeper in the PIC results. A steeper electric field yields a larger Hall current and electrons are heated. As a result of the larger electron temperature, the ion number density increases and the neutral number density becomes smaller in comparison to the hybrid-Vlasov results.

\section{Thruster performance}

The thruster efficiency, $\eta$, thrust, $T$, and specific impulse, $I_{s p}$, are calculated from

$$
\begin{aligned}
\eta & =\frac{\dot{m} v_{\text {exit }}^{2}}{2 I_{d} V_{d}} \\
T & =\dot{m} v_{\text {exit }} \\
I_{s p} & =\frac{T}{\dot{m} g}=\frac{v_{\text {exit }}}{g}
\end{aligned}
$$

where $v_{\text {exit }}$ is the exhaust velocity at the channel exit and $g$ is the acceleration due to gravity $\left(=9.8 \mathrm{~m} / \mathrm{s}^{2}\right)$.

As shown in Table 3, the results obtained from the hybrid-Vlasov simulation are in good agreement with the hybrid-PIC results. However, the simulation results show higher specific impulse and thrust than the experimental data of Mikellides et al. ${ }^{21}$ As can be seen from Equations $16 \mathrm{~b}$ and $16 \mathrm{c}$, the exhaust velocity, or the ion mean velocity at the channel exit, is overestimated. This is because the potential boundary condition of zero is set at the channel exit in the current simulation instead of at the cathode. In the real thruster, the potential drop will extend into the plume so that the ion mean velocity accelerates in the plume as well as inside the channel. In addition, plume divergence is not included in the current one-dimensional assumption.

The numerical investigations by Garrigues et al. show that doubly charged ions contribute to the discharge current. $^{20}$ Multicharged ions may also affect the exhaust velocity since they accelerate faster than singly charged ions. However, in the current hybrid-Vlasov simulation, only singly charged ions are included. 
Table 3. Thruster performance

\begin{tabular}{|c|cc|c|c|}
\hline & \multicolumn{2}{|c|}{ Hybrid-Vlasov } & Hybrid-PIC & Experiment \\
& without CEX & with CEX & & \\
\hline Mean discharge current & $4.29 \mathrm{~A}$ & $4.28 \mathrm{~A}$ & $4.34 \mathrm{~A}$ & $4.5 \mathrm{~A}$ \\
Efficiency & 0.656 & 0.651 & 0.60 & 0.5 \\
Thrust & $91.8 \mathrm{mN}$ & $91.5 \mathrm{mN}$ & $88.4 \mathrm{mN}$ & $80 \mathrm{mN}$ \\
Specific impulse & $1870 \mathrm{~s}$ & $1860 \mathrm{~s}$ & $1800 \mathrm{~s}$ & $1600 \mathrm{~s}$ \\
\hline
\end{tabular}

\section{Charge exchange collisions}

For the current simulation, the effect of charge exchange collisions does not significantly affect the overall thruster performance which is shown in Table 3. However, the difference can be seen in the VDFs for heavy species. Figure 3(a) shows the energy distribution functions (EDFs) of the ions at the channel exit compared with the measurements of Bareilles et al. ${ }^{11}$ The measured IEDF is narrower than the simulation results mainly due to the dynamic effects. Depending on the amplitude of the discharge oscillations, the ion distribution functions shift back and forth. This suggests that the current electron fluid model creates plasma oscillations that are too large in comparison with the experimental data. The effect of charge exchange collisions is small yet can be seen in the IEDF. Since fast neutral atoms are generated due to CEX collisions, the population of the ions generated via ionization has a larger tail in the high velocity region. Additionally, the VDFs of neutral atoms are shown in Figure 3(b). Although the magnitude of the high velocity tail is small compared to the bulk VDF, the fast neutral atoms generated due to CEX collisions are captured well without statistical noise.

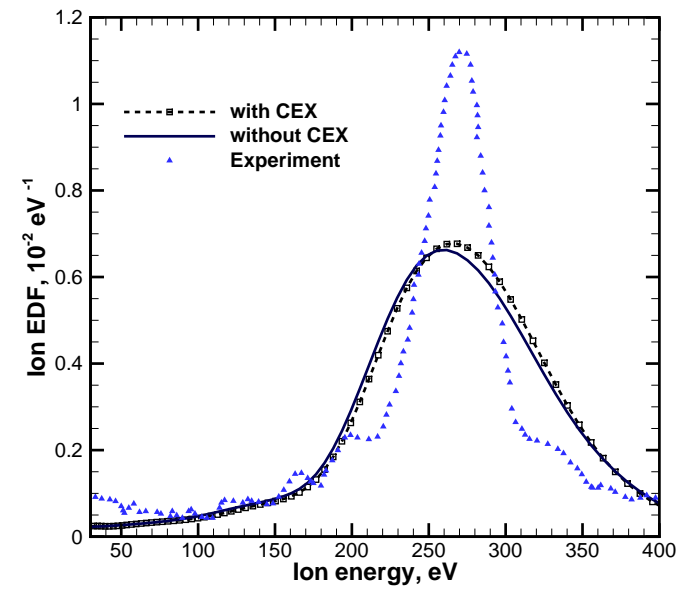

(a) Ion EDFs

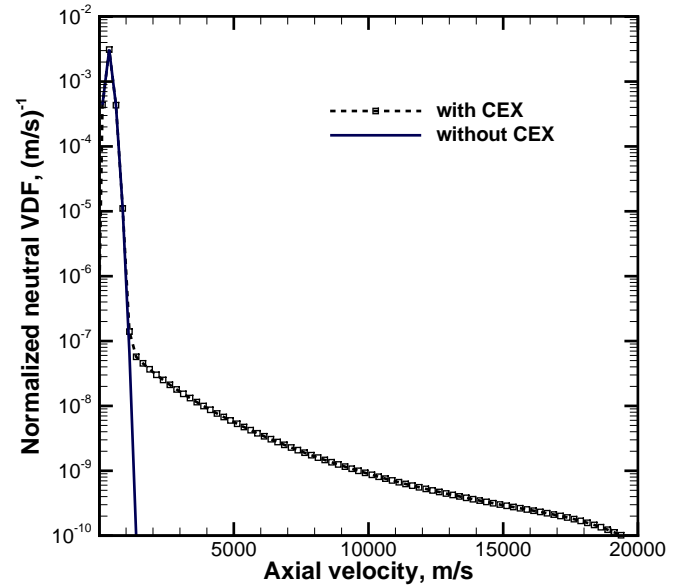

(b) Neutral VDFs

Figure 3. Distribution functions at the channel exit

\section{B. Plasma oscillations}

Plasma oscillations are found in the unsteady calculation. Low frequency oscillations, often referred to as the breathing mode, occur due to the depletion through ionization of neutral atoms. The oscillation of discharge current is shown in Figure 4. Although the plasma oscillations depend not only on the ion transport but also on the electron transport, the results from the hybrid-Vlasov simulation agree well with the measured data. ${ }^{21}$ The evolution of the macroscopic quantities obtained from the VDFs is shown.

Here, six temporal points are chosen to visualize the evolution of the number densities and mean velocities of ions as shown in Figure 5. At the maximum peak of discharge current (point 1), the plasma density is 


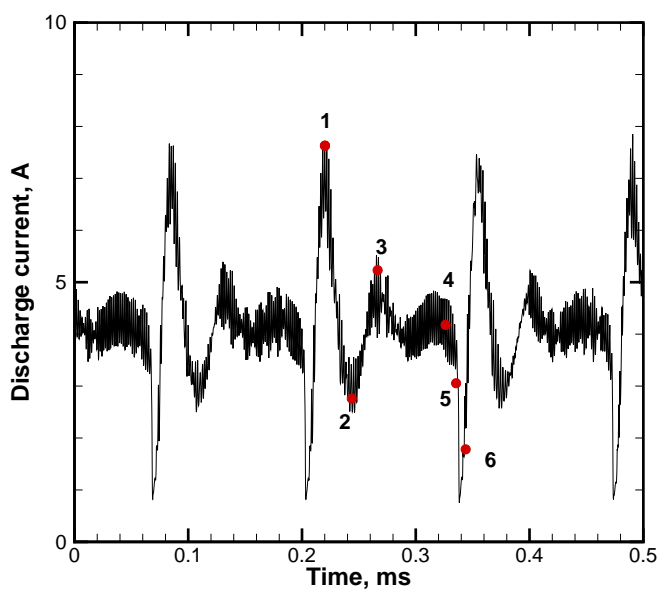

(a) Hybrid-Vlasov simulation

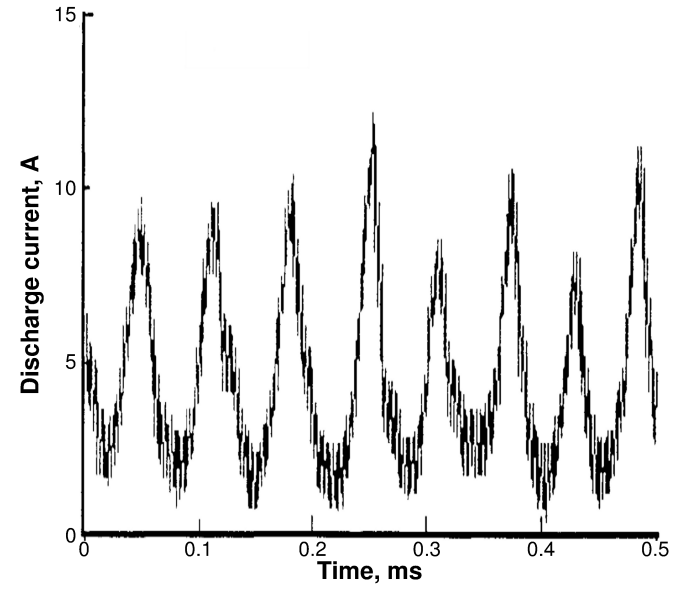

(b) Experiment in NASA/GRC

Figure 4. Discharge current oscillation: In Figure 4(a), Point 1: $220 \mu \mathrm{s}, 2: 247 \mu \mathrm{s}, 3: 266 \mu \mathrm{s}, 4: 329 \mu \mathrm{s}, 5: 338$ $\mu \mathrm{s}$, 6: $342 \mu \mathrm{s}$. Figure 4(b): Reproduced from Ref. 21

high throughout the channel so that the ion current is the largest and hence the discharge current is largest. The discharge current is smaller due to the smaller ion number densities at point 2 in comparison to point 1. At point 3 , the ion number densities in the ionization region increase again. Additionally, from point 1 to 3 , the ion number densities decrease and ions are accelerated in the diffusion region. At points 4, 5, and 6 where the old bulk plasma is being pushed out, $\mathrm{f}$ bulk plasma is generated inside the channel and goes back to point 1 . The results indicate that the anode region plays an important role in the breathing mode suggesting that the modeling of anode region is important. Recall that the anode sheath and the thermal conductivity are neglected in the current simulation.

In the operation of a Hall thruster, the breathing mode is one of the low frequency modes due to an ionization interaction between ions and neutral atoms. Two types of ionization oscillations are observed in the hybrid-Vlasov results. The first one involves the new bulk plasma being generated in the diffusion region (from point 4 to 6 ) that pushes the old bulk plasma out of the channel. Although being less obvious than the previous one, the second mechanism can be seen in the ionization region from the decrease in ion number densities from point 1 to 2 as well as the increase between points 2 and 3 . This suggests an ionization cycle from point 1 to point 3 . If we consider that the transition from point 3 to 4 is a quasi-steady behavior, the breathing mode frequency is estimated to be $14 \mathrm{kHz}$ in the hybrid-Vlasov simulation.

A simple physical model for breathing mode oscillations is proposed by Fife: ${ }^{22}$

$$
f_{B}=\frac{\sqrt{V_{i} V_{n}}}{2 \pi L_{i}}
$$

where $f_{B}$ is the breathing mode frequency, $V_{i}$ and $V_{n}$ are the characteristic velocities of ions and neutral atoms, respectively, and $L_{i}$ is the characteristic length of the ionization region. For $V_{i}=18,000 \mathrm{~m} / \mathrm{s}$, $V_{n}=173 \mathrm{~m} / \mathrm{s}$, and $L_{i}=0.02 \mathrm{~m}$, the breathing mode frequency from Equation 17 is $f_{B}=15 \mathrm{kHz}$. The experiment of Mikellides et al. shows a breathing mode frequency of $17 \mathrm{kHz} .{ }^{21}$ Although the structure of discharge oscillations observed in the hybrid-Vlasov results does not completely agree with the experiment, the breathing mode frequency obtained from the simulation is in good agreement with the theory and experiment.

The first type of breathing mode oscillations is due to ionization in the diffusion region rather than in the ionization region. Its mechanism is due to the diffusion region being filled with neutral atoms that are injected from the anode. One period of this cycle is $140 \mu \mathrm{s}$ from the simulation results. This corresponds to 

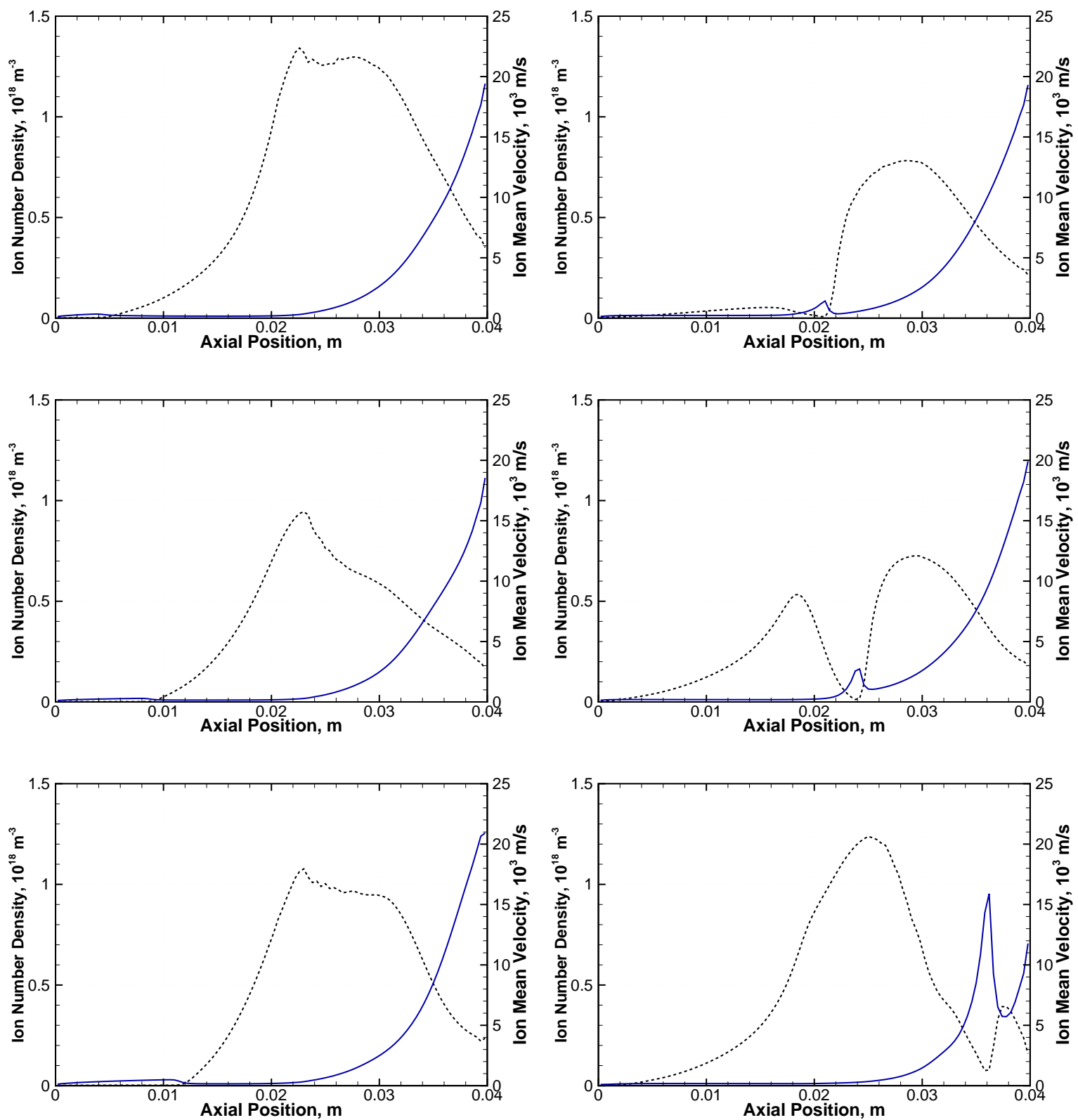

Figure 5. Evolution of the number density and mean velocity of ions. Black dashed lines: ion number density (left axis), Blue solid lines: ion mean velocity (right axis). Left column: Points 1, 2, 3 (from top to bottom), Right column: Points 4, 5, 6 (from top to bottom)

the transit time of neutral atoms which is given by

$$
\tau_{n}=\frac{L_{i}}{V_{n}}
$$

Using the values above, this gives $\tau_{n}=115 \mu \mathrm{s}$ and agrees with the time needed to fill the channel with neutral atoms in the simulation.

\section{Statistical noise in VDFs}

One of our primary goals is to investigate the capability of the Vlasov simulation in comparison with the PIC simulation. It is shown in Section A that the hybrid-PIC simulation developed by Boeuf and Garrigues 
provides similar results to our hybrid-Vlasov simulation. However, differences in the two simulations can be seen in the VDFs.

Point 4 in Figure 4 where the discharge current starts decreasing to its minimum is chosen to compare the results obtained from the two simulations. Figure 6 shows the instantaneous plasma properties and VDFs of the hybrid-Vlasov and hybrid-PIC simulations. Identical cell sizes are used for the discretization of phase space and the VDFs are obtained from the location and velocity of each macroparticle for the hybrid-PIC results.

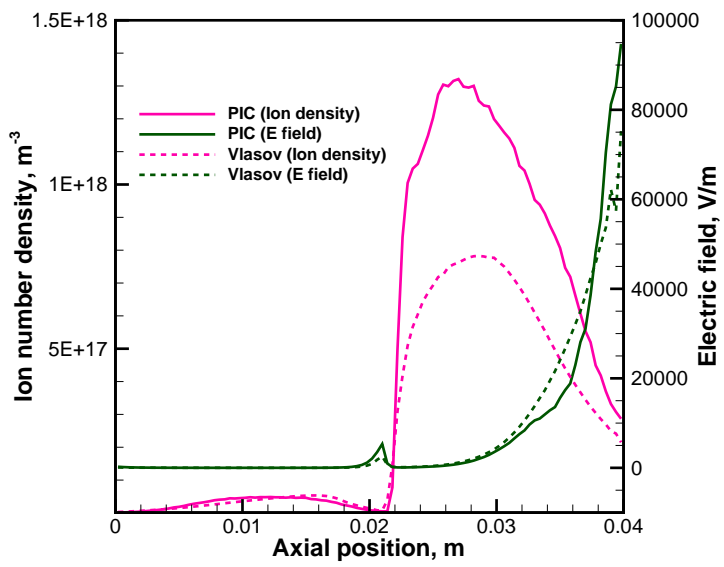

(a) Ion number density and electric field

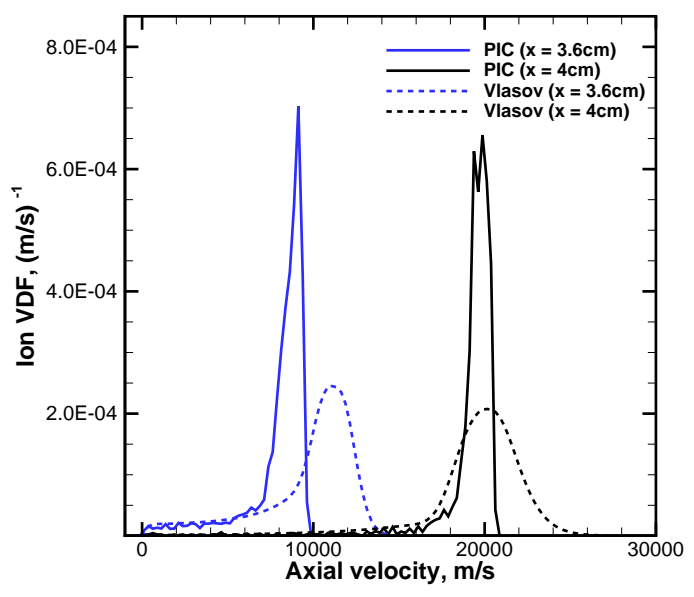

(b) Normalized ion VDFs at locations $3.6 \mathrm{~cm}$ and $4 \mathrm{~cm}$ away from the anode (4cm: the channel exit)

Figure 6. Instantaneous plasma parameters and ion VDFs at point 5 in Figure 4

As shown in Figure 6(a), the plasma parameters agree well in the diffusion region but the ion number densities obtained from the hybrid-Vlasov simulation are smaller than the hybrid-PIC results. These results are consistent with the time averaged results that the hybrid-Vlasov simulation gives smaller electron temperatures and hence smaller ion number densities in the ionization and acceleration regions as shown in Figure 2. Figure 6(b) shows non-Maxwellian VDFs of ions obtained from both simulations. The peaks of the VDFs are located at different axial velocities depending on how much the ions are accelerated due to the electric field. At $3.6 \mathrm{~cm}$ away from the anode, since a large electric field is generated for the hybrid-Vlasov simulation, the ion VDFs shift in the positive direction. The most probable velocities of the VDFs at the channel exit are approximately $20,000 \mathrm{~m} / \mathrm{s}$ for both simulations.

The VDFs obtained from the Vlasov simulation are very smooth and contain no statistical noise. However, the use of the bounded upwind scheme may cause broadening of the VDFs. For instance, the high energy tail of the VDFs is well resolved in the Vlasov simulation but it may be due to the numerical dissipation of the first-order upwind scheme used in the cells where numerical oscillations occur. Since numerical schemes with low order of accuracy are dissipative, the accuracy of the numerical schemes used to solve the Vlasov equation may be important.

On the other hand, the PIC results show statistical noise in low density regions such as the low velocity tail of VDFs and the near anode region. Careful treatment is required in the near-anode region where there are some cells that have no particles for the PIC simulation. In order to avoid zero number density, the hybrid-PIC simulation employed an offset for ion number densities of $10^{15} \mathrm{~m}^{-3}$. Otherwise, macroscopic parameters such as number density and mean velocity should be calculated by averaging them over some finite sampling time steps.

\section{Collision models}

A different collision model based on that developed by Fife ${ }^{22}$ is used and compared to the one that is proposed by Boeuf and Garrigues. Here, the Boeuf and Garrigues' model is denoted case A and the Fife model is case 
B. Different models for the inelastic cross sections are used in these two models.

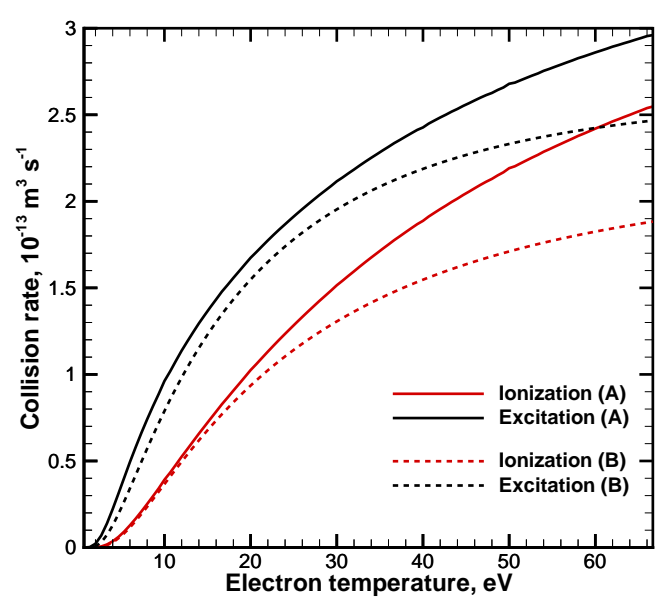

(a) Ionization and excitation rates

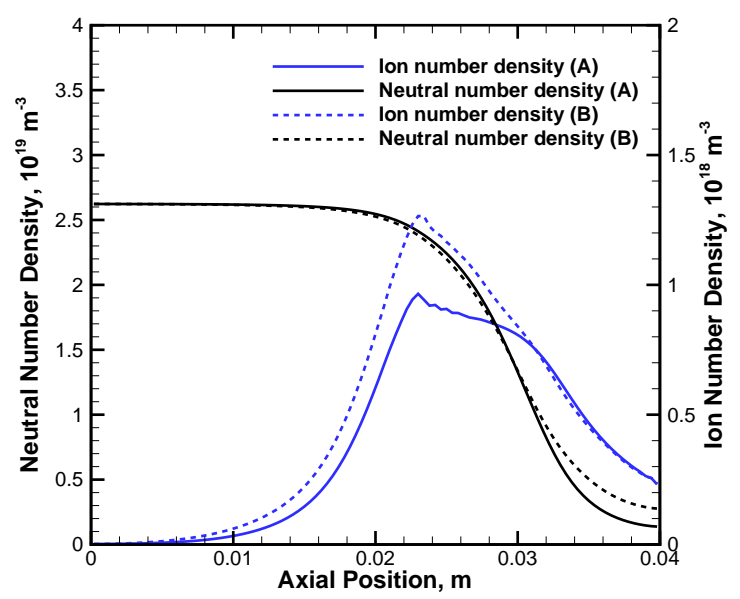

(b) Time averaged number density of heavy species

Figure 7. Results of two different cross section models (Case A: Boeuf and Garrigues and Case B: Fife)

Figure 7(a) shows the collision rates due to ionization and excitation $\left(\xi^{i}\left(T_{e}\right)\right.$ and $\xi^{e x}\left(T_{e}\right)$ in Equations 6 and 12) that are calculated by assuming a Maxwellian distribution for electrons and xenon atoms are much slower than electrons. Case A has higher ionization and excitation rates than case B at higher electron temperature. These rates contribute to the amount of ions generated via ionization as well as energy loss in the electron fluid model. In the current simulation, the electron temperature is truncated at $66 \mathrm{eV}$.

The time averaged number densities of the heavy species are shown in Figure 7(b). The ion number density is larger in the ionization region for case B than case A. Considering the lower ionization rate at high electron temperature of case B, it can be expected that case B yields smaller ion number density. However, ionization and excitation rates also contribute to energy loss in the electron energy equation. Thus, the electron temperature is larger in case B and produces more ions inside the channel. Case A, which has higher ionization and excitation rates, yields lower electron temperature and generates lower ion number density.

Table 4. Thruster performance

\begin{tabular}{|c|cc|}
\hline & Case A & Case B \\
\hline Mean discharge current & $4.29 \mathrm{~A}$ & $4.00 \mathrm{~A}$ \\
Efficiency & 0.656 & 0.631 \\
Thrust & $91.8 \mathrm{mN}$ & $86.6 \mathrm{mN}$ \\
Specific impulse & $1870 \mathrm{~s}$ & $1766 \mathrm{~s}$ \\
\hline
\end{tabular}

Thruster performance is also compared in Table 4. The exhaust velocity is smaller for case B which results in lower thrust and specific impulse in comparison with case A. This suggests that the rates of inelastic collisions such as ionization and excitation may play an important role.

\section{Conclusion}

A direct Vlasov simulation method has been developed using a bounded upwind scheme in order to preserve positivity of velocity distribution functions. Its application to a Hall thruster has been assessed. The results from the hybrid-Vlasov simulation show good agreement with experiments. In comparison to the hybrid-PIC simulation, the resolution of VDFs has significantly improved due to the direct calculation of VDFs by solving the Vlasov equation coupled with collision terms. The new kinetic approach provides an 
alternative to particle simulations that contain statistical noise even when the number of macroparticles is large.

This model will be further investigated through consideration of higher-fidelity collision models and inclusion of double-charge ionization. Additionally, the accuracy of the numerical scheme used in the Vlasov simulation will be improved so that numerical dissipation for hyperbolic PDEs can be reduced. In order to address the main disadvantage of a direct Vlasov simulation, which is the computational cost due to full discretization of spatial and velocity space, numerical techniques such as adaptive mesh refinement and parallelizing can be employed.

\section{Acknowledgments}

The authors gratefully acknowledge the financial support provided by the U.S. Department of Energy Office of Science, Fusion Energy Sciences Program, Grant DE-SC0001939, and the Air Force Research Laboratory Grant F9550-09-1-0695. K. Hara also gratefully acknowledges the financial support of the Japanese Student Services Organization.

\section{References}

${ }^{1}$ Ahedo, E., Martinez-Cerezo, P., and Martinez-Sanchez, M., "One-dimensional model of the plasma flow in a Hall Thruster," Physics of Plasma, Vol. 8, No. 6, June 2001, pp. 3058-3068.

${ }^{2}$ Keidar, M., Boyd, I. D., and Beilis, I. I., "Plasma flow and plasma-wall transition in Hall thruster channel," Physics of Plasma, Vol. 8, No. 12, December 2001, pp. 5315-5322.

${ }^{3}$ Hofer, R. R., Katz, I., Mikellides, I. G., Goebel, D. M., Jameson, K. K., Sullivan, R. M., and Johnson, L. K., "Efficacy of Electron Mobility Models in Hybrid-PIC Hall Thruster Simulations," AIAA 2008-4924, Hartford, CT, July 2008.

${ }^{4}$ Taccogna, F., Schneider, R., Longo, S., and Capitelli, M., "Kinetic simulations of a plasma thrusters," Plasma Sources Sci. Technol., Vol. 17, 2008, pp. 024003.

${ }^{5}$ Kolobov, V. I. and Arslanbekov, R. R., "Towards Adaptive Kinetic-Fluid Simulations of Weakly Ionized Plasmas," Journal of Computational Physics, Vol. 231, No. 3, 2011, pp. 839-869.

${ }^{6}$ Garrigues, L., Heron, A., Adam, J. C., and Boeuf, J. P., "Hybrid and particle-in-cell models of a stationary plasma thruster," Plasma Sources Sci. Technol., Vol. 9, No. 2, February 2000, pp. 219-226.

${ }^{7}$ Boeuf, J. P. and Garrigues, L., "Low Frequency Oscillations in a Stationary Plasma Thruster," Journal of Applied Physics, Vol. 84, No. 7, 1998, pp. 3541-3554.

${ }^{8}$ Puech, V. and Mizzi, S., "Collision Cross Sections and Transport Parameters in Neon and Xenon," J. Phys. D: Appl. Phys., Vol. 24, 1991, pp. 1974-1985.

${ }^{9}$ Pullins, S., Chiu, Y.-H., Levandier, D. J., and Dressler, R. A., "Ion Dynamics in Hall Effect and Ion Thrusters: $X e^{+}+$ Xe Symmetric Charge Transfer," AIAA 2000-0603, Reno, NV, January 2000.

${ }^{10} \mathrm{Koo}$, J. W. and Boyd, I. D., "Modeling of anomalous electron mobility in Hall thrusters," Physics of Plasma, Vol. 13, No. 033501, 2006.

${ }^{11}$ Bareilles, J., Hagelaar, G. J. M., Garrigues, L., Boniface, C., Boeuf, J. P., and Gascon, N., "Critical assessment of a two-dimensional hybrid Hall thruster model: Comparisons with experiments," Physics of Plasmas, Vol. 11, No. 6, June 2004, pp. 3035-3046.

${ }^{12}$ Arber, T. D. and Vann, R. G. L., "A Critical Comparison of Eulerian-Grid-Based Vlasov Solvers," Journal of Computational Physics, Vol. 180, 2002, pp. 339-357.

${ }^{13}$ Shoucri, M., Numerical Solution of Hyperbolic Differential Equations, Nova Science Publishers, 2008.

${ }^{14}$ Buchner, J., Vlasov-code simulation, Advanced Methods for Space Simulations, 2007, pp. 23-46.

${ }^{15}$ Banks, J. W. and Hittinger, J. A. F., "A New Class of Nonlinear Finite-Volume Methods for Vlasov Simulation," IEEE Transactions on Plasma Science, Vol. 38, No. 9, Sept. 2010, pp. 2198-2207.

${ }^{16}$ Colella, P., "Multidimensional upwind methods for hyperbolic conservation las," Journal of Computational Physics, Vol. 87, 1991, pp. 171-200.

${ }^{17}$ Choueiri, E. Y., "Plasma Oscillations in Hall Thrusters," Physics of Plasma, Vol. 8, 2001, pp. 1411-1426.

${ }^{18}$ Herrmann, M., Blanquart, G., and Raman, V., "Flux Corrected Finite Volume Scheme for Preserving Scalar Boundedness in Reacting Large-Eddy Simulations," AIAA Journal, Vol. 44, No. 12, December 2006, pp. 2879-2886.

${ }^{19}$ Reid, B. M., The Influence of Neutral Flow Ratein the Operation of Hall Thrusters, Ph.D. thesis, University of Michigan, 2009.

${ }^{20}$ Garrigues, L., Boyd, I. D., and Boeuf, J. P., "Computation of Hall Thruster Performance," Journal of Propulsion and Power, Vol. 17, No. 4, July 2001, pp. 772-779.

${ }^{21}$ Mikellides, I. G., Katz, I., Mandell, M. J., and Snyder, J. S., "A 1-D Model of the Hall-Effect Thruster with an Exhaust Region," AIAA 2001-3505, Salt Lake City, UT, July 2001.

${ }^{22}$ Fife, J. M., Hybrid-PIC Modeling and Electrostatic Probe Survey of Hall Thrusters, Ph.D. thesis, MIT, 1998. 